\title{
El dogma extensionalista de las aproximaciones veritativo-condicionales del significado
}

\section{The extensionalist dogma of truth-conditional approaches to meaning}

\author{
LETICIA SANTOS VARONA \\ Universidad de Valladolid
}

Recibido: 21/11/16 Aceptado: 19/12/16

\section{RESUMEN}

La concepción veritativo-condicional del significado es una explicación de la relación representacional del lenguaje como una relación entre el lenguaje y la realidad. Se ha hecho uso de la noción de condiciones de verdad para expresar una conexión directa entre los conceptos de significado como referencia y verdad, sobre la base del presupuesto de que los lenguajes naturales se comportan como lenguajes formales interpretados. Se presentarán argumentos sobre la imposibilidad de contar con una teoría de la referencia que sustente la explicación veritativo-condicional. La relación representacional lenguaje-realidad falla en la explicación del significado del lenguaje natural.

\section{PALABRAS CLAVE \\ SEMÁNTICA, REFERENCIA, SIGNIFICADO, CONDICIONES DE VERDAD}

\begin{abstract}
The truth-conditional conception of meaning is an explanation of representational relation of language as a relation between language and reality. The notion of truth conditions has been used to express a direct connection between the concepts of meaning as reference and truth, based on the assumption that natural languages behave as interpreted formal languages. Arguments
\end{abstract}


will be presented about the impossibility of having a theory of reference that supports the truthconditional explanation. The representational relation language-reality fails in the explanation of the meaning of natural language.

KEYWORDS

SEMANTICS, REFERENCE, MEANING, TRUTH CONDITIONS

\section{EL SUPUESTO VERITATIVO-CONDICIONAL}

La búsqueda de la objetividad tras el idealismo llevó a las filosofías analítica y postanalítica a desterrar el término «idea», en su vieja acepción representacional, eliminando el pensamiento de la relación de representación semántica esquematizada en el triángulo semiótico. La relación representacional se restringió a la relación lenguaje-realidad intentando mostrar cómo las expresiones lingüísticas representan lo real. La semántica clásica explica esta relación utilizando fundamentalmente tres nociones: oración, referencia y verdad.

En primer lugar, la semántica clásica convierte la oración en el vehículo primario de la representación y buena parte de la tarea semántica ha estado centrada en el estudio de la forma lógica de las oraciones. En segundo lugar, el significado se explica fundamentalmente a partir del concepto de referencia de forma que, a grandes rasgos, una oración representa/refiere/significa objetos o hechos del mundo, de modo que conocer su significado es conocer los objetos o hechos a que refiere. En tercer lugar, se postula una relación directa entre la referencia y la verdad. El significado se produce cuando asociamos oraciones con el mundo y, en la medida en que se da de forma correcta esta asociación, contamos con expresiones lingüísticas verdaderas. Si uno se fija en la forma en que se caracterizó el significado unas líneas más arriba es fácil ver la conexión entre la referencia y la verdad que hace entrar en juego al concepto de condiciones de verdad: una oración refiere/significa objetos o hechos del mundo, de modo que conocer su significado es conocer los objetos o hechos a que refiere, es decir, las condiciones que debe tener el mundo bajo las que la oración es verdadera. Desde esta conexión deriva en último término la idea de que la semántica de las oraciones se expresa en términos de condiciones de verdad.

La semántica clásica asume cuatro tesis: 1) el lenguaje representa por sí mismo la realidad y tal representación se muestra en la forma lógica de las oraciones, 2) la verdad es una propiedad atribuible a las expresiones lingüísticas (concretamente a las oraciones o a las proferencias de las mismas, según nos situemos en una u otra teoría), 3) la determinación de las condiciones de verdad de las expresiones es el criterio de su significatividad pues conocer éstas contribuye a saber qué representan y, por tanto, su significado y 4) el contenido veritativo-condicional está determinado por la relación de referencia en 
la medida en que se necesita el conocimiento del mundo para determinar las condiciones de verdad.

Las aproximaciones veritativo-condicionales del significado han adoptado esta explicación como una forma de ofrecer una explicación recursiva y sistemática del significado al modo de la semántica tarskiana de los lenguajes formales, pues bajo éstas se esconde el presupuesto según el cual el lenguaje natural es una extensión del lenguaje formal. La explicación veritativo-condicional parte de las tesis de Chomsky y de Montague (Bach, 1989). Según la Tesis de Chomsky los lenguajes naturales pueden describirse como sistemas formales; a lo que la Tesis de Montague añade que los lenguajes naturales pueden ser tratados como lenguajes formales interpretados.

En resumen, cabe destacar dos premisas que han marcado los desarrollos semánticos hasta la actualidad: 1) las condiciones de verdad son el criterio del significado de las oraciones y 2) el lenguaje natural es una extensión del lenguaje formal y, como tal, las herramientas de la lógica son las herramientas para el estudio del lenguaje natural capaces de darnos una teoría recursiva del significado.

Ahora bien, la piedra angular de la concepción veritativo-condicional es la referencia por dos motivos. Por un lado, el significado se caracteriza como una asociación de expresiones y objetos y, por otro, porque lo que contribuye para determinar si una oración es verdadera son las referencias de los términos constituyentes de la oración y, por tanto, el valor de verdad de una oración depende de las referencias de sus expresiones componentes (más su articulación lógica). Este carácter decisivo de la referencia constituye al mismo tiempo, como se verá, el motivo para el rechazo de dichas teorías.

\section{EL HUECO ENTRE EL LENGUAJE NATURAL Y LOS LENGUAJES FORMALES}

En esta sección se revisará el presupuesto que asume que el lenguaje natural es una extensión del lenguaje formal concluyendo el rechazo del supuesto veritativo-condicional.

\section{1. AMBIGÜEDAD VS. PRECISIÓN}

Ambos lenguajes, natural y formal, se componen de elementos similares: un vocabulario de signos, operadores (símbolos relacionantes) y unas reglas para el uso correcto de signos y operadores. Además de estas semejanzas sintácticas, ambos son, como todo lenguaje, sistemas simbólicos con capacidad representacional y, por ello, tienen una dimensión semántica, una forma determinada en la que se relacionan los símbolos con aquello que simbolizan. Sin embargo, presentan una diferencia crucial: el lenguaje natural es fundamentalmente ambiguo, mientras el lenguaje formal es esencialmente preciso. 
El lenguaje formal es un lenguaje artificialmente creado como herramienta de análisis científico en el que representar ciertos contenidos para estudiar sus características. La utilidad de estos lenguajes se encuentra en su estructura sintáctica. Los lenguajes formales como sistemas lógicos son fundamentalmente sintácticos de forma que abstraen los contenidos de los enunciados. Esto posibilita representar en un mismo lenguaje distintas realidades con la garantía de que tales sistemas preservan la verdad pues son estructuras donde se garantiza la inferencia válida. Lo que importa en un sistema lógico es si las fórmulas que se deducen del sistema son verdaderas. Esto se consigue regimentando la sintaxis a través de definiciones recursivas de los elementos y de las relaciones (siempre deductivas) del sistema de modo que haya transparencia sintáctica. De esta forma, los símbolos del lenguaje así como la forma en que pueden combinarse para crear símbolos complejos y para justificar la inferencia de unos a otros están especificados.

Sin embargo, para que un sistema lógico tenga aplicabilidad y constituya realmente un lenguaje debe contar no sólo con una estructura sintáctica, sino además con una semántica. La semántica explica la relación entre el lenguaje y la realidad sujeta a análisis a partir de nociones incorporadas desde la Teoría de conjuntos matemática. Una forma común de hacer esto es explicar la semántica de los lenguajes formales en términos de Teoría de modelos. Dado que el significado de los símbolos no es propiamente lenguaje sino la realidad que representa y en un lenguaje formal no hay hablantes, en la Teoría de modelos se opta por una definición semántica extensional: se asume que los significados de los símbolos son sus denotaciones. La tarea semántica entonces consiste en interpretar un lenguaje (asignar denotaciones a los símbolos del lenguaje): primeramente se deben asignar denotaciones a los elementos básicos y posteriormente explicar cómo a partir de la combinación de estos se obtienen los significados de las fórmulas complejas.

Para ello son necesarias dos cosas. Primero, es necesario tener denotaciones. Ahora bien, dadas las características de los lenguajes formales, las denotaciones deben estar precisadas, es decir, se debe contar con un dominio de objetos sobre los que el lenguaje hable. Esta precisión implica que se conocen todos los objetos que hay en el dominio, así como todas las propiedades que estos tienen de manera que el dominio está estructurado en términos de conjuntos matemáticos. Lo que se encuentra en el dominio son exclusivamente objetos $\mathrm{y}$, por tanto, las propiedades están definidas en este sentido extensional como conjuntos de individuos (donde la pertenencia a un conjunto es una cuestión definida). Segundo, debe especificarse la relación que los objetos tienen con las expresiones del lenguaje, es decir, que debe estar clara la forma en que se conectan los símbolos con el dominio. Esto se consigue a través del concepto matemático de función. Una función no es más que una relación con una 
característica esencial: es una relación unívoca entre dos conjuntos de forma que a cada elemento del primer conjunto le corresponde inequívocamente un elemento del segundo. Describir la relación semántica a través de funciones da como resultado un sistema semánticamente transparente puesto que no sólo la relación entre estos es inequívoca sino que además a cada símbolo le corresponde un elemento del dominio (Barba, 2010).

Ahora bien, la función, como objeto matemático que es, también es una expresión de cálculo (Frege, 1904). Esta característica es fácilmente visible cuando de lo que hablamos es de identificar las denotaciones (significados) de las fórmulas complejas. Para los términos básicos los valores son denotaciones del dominio (dada una función como «El lugar donde nació x» y la asignación «Delibes» a la variable, se obtiene que el valor de la función es la denotación de un lugar «Valladolid»); sin embargo, para fórmulas complejas, el valor de la función es un valor de verdad. Esto se debe a que la definición de los operadores lógicos es veritativo-condicional pues en un sistema lógico lo que interesa es garantizar la inferencia válida. Así, se asume desde Frege (1892) que la denotación de una fórmula compleja es su valor de verdad, resultado del modo de combinación veritativo-condicional de las partes simples que la integran. Esta idea se mantiene en la Teoría de modelos según la cual los términos (constantes y variables) denotan individuos, los predicados denotan conjuntos de individuos y las oraciones valores de verdad. La Semántica formal se construye en definitiva a partir fundamentalmente de denotaciones, como valores semánticos, y funciones (Portner, 2005).

Más allá de los detalles técnicos, lo que interesa es mostrar tres conclusiones: 1) en un lenguaje formal sintaxis y semántica están en correspondencia ${ }^{1}$ y ésta es transparente, de modo que no cabe ambigüedad, 2) lo que aporta el significado no es lenguaje sino ontología, que debe darse previamente y definirse matemáticamente mediante una asignación precisa y unívoca ${ }^{2}$ y 3 ) hay

1 La correspondencia se construye bajo lo que se ha llamado con la teoría de Montague la hipótesis regla por regla. Según ésta debe haber una regla semántica por cada regla sintáctica (o categoría sintáctica). De esta forma la composicionalidad que restringe la sintaxis se mantiene en la semántica (Dowty et al. 1981).

2 Si bien es cierto que la semántica de mundos posibles vinculada a la Teoría de modelos incluye intensiones, no debe caerse en el error de atribuir al concepto de intensión un carácter que no tiene. Las intensiones, como recurso técnico, no son más que conjuntos de denotaciones (el conjunto de las denotaciones que una expresión tiene en todos los mundos posibles). Por otra parte, dado que para tener una intensión se debe tener la denotación de la expresión de la que es intensión en cada mundo (y tiempo), necesitamos tener las denotaciones de los nombres y predicados que están involucrados en la expresión. Las intensiones son estrictamente hablando definibles extensionalmente por lo que el modelo de explicación continúa en la línea de la extensionalidad (Bach, 1989). 
una conexión directa entre la verdad y el significado pues el significado es, en último término, una función que conecta expresiones con denotaciones y la verdad, entendida como validez semántica, consiste en la correspondencia entre los símbolos y el dominio; así, la verdad está expresada en la función de asignación semántica. En consecuencia, el lenguaje formal se caracteriza por ser un lenguaje esencialmente preciso y transparente en el que se sabe qué es tanto una fórmula bien formada como de qué cosas habla el lenguaje.

El lenguaje natural en cambio está dominado por la ambigüedad. Los hablantes tenemos libertad expresiva para hacer la comunicación efectiva y esto produce que la ambigüedad en el lenguaje natural sea un fenómeno generalizado que se puede encontrar tanto en la estructura sintáctica como en la semántica. En relación a la sintaxis, tal es la libertad que podemos violar las reglas sintácticas en ciertos ámbitos de comunicación. A menudo en las conversaciones cotidianas emitimos palabras aisladas o frases sintácticamente incompletas, en el sentido en que no se sigue la estructura especificada por las reglas de la gramática, pues uno puede suprimir palabras si con ello gana en eficacia comunicativa. Incluso, la ambigüedad se da también en el caso de oraciones sintácticamente correctas. Esto ocurre en oraciones que pueden entenderse como formadas por dos estructuras sintácticas diferentes de forma que presentan una ambigüedad estructural ( Ha comprado naranjas y mandarinas grandes» puede entenderse como que ha comprado naranjas grandes y mandarinas grandes o que ha comprado naranjas de cualquier tamaño y mandarinas grandes).

Pese a que este tipo de ambigüedad se da con mucha frecuencia, es quizá el fenómeno de la ambigüedad semántica el más acusado dentro del lenguaje natural. A través del lenguaje natural podemos expresar una infinidad de conceptos, objetos y hechos del mundo con una amplia variedad de formas de expresión de manera que la ambigüedad léxica, que incluye fenómenos como la polisemia o la homonimia, es un fenómeno que se da con alta frecuencia. Por otra parte, podemos utilizar el lenguaje de una forma más o menos convencional, utilizar recursos figurativos como la metáfora e, incluso, dentro de nuestra libertad expresiva podemos encontrar paradojas, como es el caso de la conocida oración «Soy un mentiroso». Además, es posible encontrar distintos tipos de expresiones cuyo significado es intrínsecamente vago, como el caso de los adjetivos, adverbios o términos que expresan conceptos abstractos. En relación a estos una explicación de la vaguedad de los adjetivos servirá para explicar a su vez la ambigüedad de otros términos. Es usual mencionar como un caso relevante de ambigüedad semántica los adjetivos gradables como «calvo» cuya definición implica la conocida paradoja de sorites pues no hay un punto exacto de demarcación entre aquello que entraría dentro del concepto de calvicie. Igualmente, una gran parte de los sustantivos y adverbios que son derivaciones de adjetivos arrastran el mismo tipo de ambigüedad. Es fácil ver como 
para sustantivos comunes como «riqueza», «tamaño» o «altura», entre otros, o para adverbios como «lentamente» tampoco contamos con un punto preciso de demarcación para la decisión sobre qué entidades caen bajo cada concepto. En el caso de los adverbios que no derivan de adjetivos, estos implican matices temporales, espaciales o modales, entre otros, de una forma indefinida (piénsese en la indefinición del matiz de cantidad del adverbio en la oración «Llovió mucho»). En el fondo, una gran parte de las expresiones de nuestro lenguaje involucran la vaguedad que envuelve la paradoja de sorites. No hay un único criterio para decidir cuándo uno es guapo, alto, rico, generoso ni para decidir cuándo uno está corriendo lentamente o cuánto es llover mucho. La semántica del lenguaje natural es por naturaleza vaga y las relaciones (semánticas) que se establecen entre los símbolos y los objetos no parecen unívocas.

Hay también una ambigüedad derivacional que afecta de forma simultánea a la sintaxis y a la semántica pues implica el concepto de forma lógica derivado de la Semántica formal. La forma lógica hace referencia a la estructura relevante para la representación semántica de contenidos (Creswell, 2006; Ludwing, 2012). En un lenguaje formal ésta se muestra en la sintaxis, sin embargo en los lenguajes naturales el asunto es un poco más complejo. Si el lenguaje natural tiene una forma lógica asociada a la sintaxis y a la semántica, ejemplos como el que sigue muestran dos cosas: que la forma lógica en el lenguaje natural es distinta a la sintaxis (superficial) y que el lenguaje es ambiguo en la derivación de la misma. En la oración «Ralph believes that someone is a spy» (Quine, 1956, p.178) se pueden derivar dos formas lógicas distintas según se tome una lectura de dicto o de re. En la lectura de re Ralph cree que alguien concreto es un espía, mientras en la lectura de dicto simplemente cree que hay espías aunque no sabe quiénes son. Ambas son formas lógicas diferentes para una misma oración con la misma estructura sintáctica (superficial) y, por tanto, la oración es ambigua en este último sentido.

\section{2. SEMÁNTICA VS. LÓGICA}

Para la Semántica formal las diferencias entre ambos lenguajes son resolubles desde el uso del aparto formal. A primera vista parece posible un tratamiento del lenguaje natural en términos de lenguajes formales puesto que ambos son lenguajes en los que hay símbolos que representan cosas y la Teoría de modelos ofrece la posibilidad de formalizar este tipo de representación semántica. La idea básica entonces es especificar los símbolos y las reglas sintácticas y asignar significados a las expresiones de forma reglada. Así, la tarea de la Semántica formal ha estado encaminada a construir sistemas lógicos capaces de representar el lenguaje natural. 
A pesar de que el desarrollo del aparto lógico ha conseguido representar una buena cantidad de expresiones del lenguaje natural ${ }^{3}$, aún persiste un hueco entre ambos lenguajes. La cuestión no es cuántos tipos de expresiones podemos representar formalmente sino que lo que hace que tal hueco sea insalvable es que la representación formal en términos de semántica lógica del lenguaje natural no puede dar cuenta de la naturalidad de nuestro lenguaje. Los argumentos para la defensa de esta idea siguen dos líneas. La primera está en íntima relación con lo que Pietroski (2005) ha denominado el dogma extensionalista pues los argumentos que aquí se presentan constituyen un complemento para apoyar la existencia de tal dogma asumido por las semánticas veritativo-condicionales. La segunda tiene que ver con la confusión entre propiedades lógicas y semánticas.

Para explicar la primera línea es necesario recordar dos requisitos fundamentales de la Teoría de modelos: el significado está vinculado con la denotación y la atribución semántica se lleva a cabo a través de funciones. Si uno quiere dar un tratamiento formal de la semántica natural en términos de modelos debe especificar el dominio que está tras la interpretación semántica, es decir, debe especificar la información sobre la que habla el lenguaje. Sin embargo, no es posible contar con una teoría de la referencia para el lenguaje natural que cumpla los requisitos exigidos.

En primer lugar, hay muchas expresiones que carecen de referencia extensional definida: podemos encontrar oraciones con expresiones que denotan entidades de ficción cuya contraparte extensional es inexistente, como, por ejemplo, aquellas que integran unicornios, hadas o duendes; algunas expresiones denotan entidades epistémicamente inaccesibles, como las que hablan de entidades futuras (Peacocke, 2000) o como «The god Apollo» en «The god Apollo was worshipped in the island of Delos» (Seuren, 2009, p. 218); es fácil ver el problema que plantea determinar una referencia extensional para expresiones no reductibles a objetos físicos como «la Quinta sinfonía de Beethoven» (Horgan, 1986) o para términos teóricos como Big Bang; por último, las palabras abstractas carecen de una referencia extensional clara como ocurre con «lan-

3 Montague consiguió resolver la diferencia en el comportamiento de ciertas expresiones como los sintagmas nominales o los cuantificadores del lenguaje natural respecto del lenguaje formal introduciendo herramientas como el operador lambda o los cuantificadores generalizados, así como resolvió el problema del cambio de valor de verdad de las oraciones oblicuas con la Teoría de tipos intensional. Sin embargo, había expresiones del lenguaje natural que la teoría no trataba (la inexistencia de plurales y de verbos más allá de la tercera persona o los significados de palabras abstractas entre otros). Nuevos desarrollos han conseguido dar expresión formal a éstas aumentando la estructura del modelo: la teoría de los plurales y de los términos masa de Link (1983), la introducción de escenas de Carlson (1977) y el tratamiento de las propiedades de Chierchia y Turner (1988). 
guage» en la oración «Chinese is the language of Beijing and Hong Kong, but not Melbourne» ${ }^{4}$ (Chomsky, 2000, p.130). Quizá podría decirse que todas las expresiones denotativas del lenguaje natural plantean el problema de la referencia vacía en mayor o menor agudeza (Strawson, 1952). En segundo lugar, fenómenos como la polisemia, la sinonimia o los usos figurativos muestran que la relación de referencia no es una relación simple marcada por el carácter matemático del concepto de función. En el lenguaje natural las relaciones referenciales no parecen unívocas sino más bien flexibles dado que las referencias de muchas expresiones pueden variar en función del uso y la intención comunicativa del hablante.

Determinar la referencia de la mayor parte de las expresiones con independencia de los sujetos es una tarea problemática desde un punto de visto teórico pues obliga a entrar en consideraciones metafísicas y epistemológicas. Es inevitable recordar cómo el empirismo o el constructivismo kantiano, entre otros, nos advirtieron de cómo los objetos con independencia del sujeto nos son inaccesibles y de cómo nuestra cognición construye la realidad. Russell (1918) dedicó varias páginas a la aparentemente sencilla tarea de determinar la referencia de «esta mesa»; en nuestros días, Chomsky (2000, p. 37) ha mostrado que palabras como «Londres» tomadas en un sentido extensional producen la adscripción de propiedades curiosas a dicha entidad, como que pueda ser destrozada y reconstruida posteriormente en otro lugar y tiempo sin dejar de ser Londres. Exigir denotata extensionales a los términos lingüísticos puede retrotraernos al viejo problema metafísico de la esencia: ¿La referencia de «Londres» es una localización, un área, su población? El problema del conocimiento de los objetos es un problema sin resolver de larga tradición filosófica del que uno no puede despojarse sin más asumiendo que hay objetos en el mundo, que los conocemos y que nuestras palabras los refieren como si estuvieran atados por un hilo invisible.

Más bien parece que lo que tomamos como objetos y cómo los referimos depende de factores cognitivos. Categorizamos las entidades según distintos criterios y cuando usamos las palabras para referir a dichas entidades priorizamos alguno de estos criterios en función de las perspectivas y recursos disponibles a nuestra cognición de forma que la asignación de referencias depende del entramado de información sobre el mundo, los intereses o las experiencias individuales entre otros. Así, uno puede usar «Londres» para referirse a su población o, en otras circunstancias, para referir a una extensión de terreno. Las expresiones

4 El revisor de la revista Contrastes apunta que, dado que el lenguaje hablado en la República Popular de China es el Mandarín, lo que probablemente debiera tener en mente Chomsky sería éste último cuando ofreció este ejemplo. El lector puede sustituir «Chinese» por «Mandarin» sin perjuicio para el argumento. 
del lenguaje natural están de esta forma cargadas de categorías semánticas y, en este sentido, no seleccionan sin más una entidad en el mundo. Dos conclusiones se siguen: 1) el léxico es más rico, complejo y flexible de lo que la visión derivada de la Semántica formal sugiere (Chomsky, 2000; Falkum y Vicente, 2015; Pietroski, 2005) y 2) la forma en que la experiencia se conceptualiza no parece un isomorfismo simple de la manera en que es verbalmente expresado (Bierwise y Schreuder 1992). En definitiva, la referencia en el lenguaje natural es algo muy distinto al concepto de denotación extrapolado desde los lenguajes formales (Rayo, 2013) y, por tanto, no se puede afirmar sin más que un término del lenguaje natural tiene una extensión (Putnam, 1975).

En este sentido, volviendo a la Teoría de modelos, ésta no dice cómo se determinan las denotaciones que conforman el dominio (Montague dejó esta tarea a la ciencia y a la psicología), sino que más bien dice cómo aplicar una estructura y, una vez dados los elementos, cómo son las relaciones entre ellos. La elección de los objetos que son candidatos a denotaciones es previa e independiente del sistema e implica un compromiso ontológico. Si uno quiere dar un tratamiento formal en términos de modelos de la semántica natural debe especificar el dominio que está tras la interpretación semántica y, por tanto, debe ser posible asignar una referencia para cada término; en otras palabras, solucionar el problema de la referencia vacía y de la falta de univocidad en las relaciones semánticas del lenguaje natural. No obstante, los ejemplos dados anteriormente muestran la dificultad de tal tarea pues sería necesaria una precisión metafísica que no tenemos. No es posible especificar un único dominio para el lenguaje natural en términos de denotaciones y funciones (Robinson, 2009). Lo que tenemos son distintos modelos en los que se ha llevado a cabo una decisión ontológica arbitraria sobre aquellas cosas de las que habla el lenguaje natural. Así, uno puede elegir la metafísica del lenguaje natural que considere: tomar escenas (Carlson, 1977), eventos (Davidson, 1980) o situaciones (Creswell, 1973) como siendo entidades de nuestro mundo; se pueden tomar los objetos físicos como cantidades (estructuradas) de materia (Link, 1983); los números pueden ser conjuntos o individuales como en la teoría de Montague (Bach, 1989); se puede optar por una concepción en la que los elementos del tiempo sean puntos en una línea o intervalos (Bach y Chao, 2012); se pueden aceptar clases, funciones, mundos, etc.

La cuestión entonces es la decisión sobre cuáles de estos modelos son los adecuados para la caracterización del lenguaje natural pero para ésta no hay respuesta disponible. Si queremos tomar seriamente la semántica natural desde un punto de vista extensional debemos aceptar que en lo que respecta al lenguaje natural sólo hay una ontología. Sin embargo, describir ésta no parece que pueda ser la labor de un lingüista, un lógico o un filósofo, sino más bien la labor de un dios omnisciente. Por ello afirma Bach (ibid., p. 135) que la semántica en los 
enfoques estándar es más bien una «semántica para Dios». La Semántica formal parece que intenta describir la ontología a partir de lo que puede construir a través de las herramientas lógicas disponibles. El formalista de la semántica natural es «un metafísico reformador» (Strawson, 1981, p. 608) pues la construcción de sistemas lógicos para el lenguaje natural no nos ayuda a comprender cómo hablamos sino que se conciben otras situaciones, distintas de las reales, imaginando como podríamos (o deberíamos) hablar y simplificando con ello la cuestión del significado. Esto definitivamente no es lo mismo que tener un modelo para el lenguaje natural pues para tenerlo es necesaria una teoría de la referencia que nos diga cuáles son los referentes de las expresiones y cómo es la conexión entre estos. Lo contrario no es más que un modo de hablar formal superficial. El hueco entre el lenguaje natural y el lenguaje formal se muestra insalvable sin una metafísica del lenguaje natural.

La segunda línea argumental ahonda en la justificación de esta imposibilidad sobre la base de la diferencia de hecho entre ambos lenguajes. Los enfoques veritativo-condicionales incurren en el error de confundir propiedades lógicas y semánticas. Lo que se entiende como propiedad lógica es la propiedad de transmitir la verdad garantizando la inferencia válida, mientras que propiedad semántica es la capacidad que tiene una expresión de representar algo diferente de sí misma. En los lenguajes formales referencia, significado y verdad coinciden. Una vez elegidas las denotaciones y hecha la asignación semántica en términos de funciones, la verdad de las fórmulas tiene independencia cognitiva (Haack, 1978; Quine, 1953; Seuren, 2009). Determinar la verdad de las expresiones en estos lenguajes es más bien un ejercicio de capacidades lógicas pues se cuenta con una noción de fórmula bien formada que restringe las interpretaciones, hay ausencia de vaguedad y los valores semánticos se preservan. Esto posibilita que los valores de verdad sean independientes de la realidad y de los agentes epistémicos. En este sentido, verdad y falsedad no tienen implicación epistémica o metafísica sino que son términos técnicos para representar la validez y es un asunto sin importancia cómo etiquetar las valuaciones de los enunciados. En lugar de «verdadero» $\mathrm{y}$ «falso» podría utilizarse sin perjuicio otros términos, como 1/0, pues la regimentación del sistema asegura la validez (Dowty et al., 1981; McGee, 2006). Así, podemos describir las propiedades semánticas en términos de propiedades lógicas y, por tanto, el significado veritativo-condicionalmente.

Sin embargo, los lenguajes naturales no se comportan del mismo modo. Para describir el comportamiento lógico de las oraciones (determinar sus condiciones de verdad) se necesita tener todos los contenidos referenciales y que éstos, como valores semánticos, se preserven para garantizar la propiedad lógica. No obstante, el lenguaje natural no cumple tales restricciones: 1) El fenómeno de la referencia vacía imposibilita tener todos los contenidos referenciales; 2) dado que la asignación semántica en términos de denotaciones no es unívoca 
en el lenguaje natural debido a su ambigüedad, los valores semánticos no se preservan a través de los usos lingüísticos y esto hace que no podamos contar en el lenguaje natural con nada parecido a lo que contaría como constante lógica en los lenguajes formales, necesario para poder sistematizar las implicaciones lógicas (Glanzberg, 2015; Lepore, 1983; Schiffer, 2015); 3) en el lenguaje natural no tenemos una noción de fórmula bien formada que restrinja las interpretaciones y que impida el desplazamiento de los términos en las interpretaciones sino que es posible interpretar términos de las oraciones como estando en una posición diferente de la que efectivamente están (Chomsky, 2000). Estos hechos incapacitan al lenguaje natural para cumplir la propiedad lógica de preservar la verdad. El lenguaje natural no cumple el principio de independencia cognitiva y esto hace que verdad, referencia y significado no coincidan (Seuren, 2009; Strawson, 1981; Szabó, 2012).

Al contrario que en los lenguajes formales, la semántica natural no tiene una estructura tarskiana que garantice el vínculo entre significado como denotación y verdad. Si fuera así, como dice Pietroski (2008), sería una especie de «benevolencia cósmica» la que permitiera que una simple computación reflejara el mundo independiente del lenguaje para que éste tuviera unas condiciones de satisfacción determinadas composicionalmente de una forma precisa:

One cannot just declare that an expression like set of bald linguists who often use heterological words has a compositionally determined satisfaction condition. (Pietroski ibid., p. 334).

En el modelo tarskiano los objetos que satisfacen los predicados deben estar definidos con anterioridad en un lenguaje teórico. Así, en el caso de «Pb is true iff 2 is prime» (Pietroski ibid., p. 334) podemos determinar las condiciones de satisfacción pues los números naturales pueden definirse con anterioridad de forma que, dadas ciertas convenciones, el número dos puede identificarse con un conjunto y sabemos qué es para un número «ser primo». Sin embargo, no podemos usar el concepto de satisfacción tarskiana para el lenguaje natural pues, como se ha visto, en el lenguaje natural hay un hueco entre la verdad y el lenguaje producido por la defectividad sintáctica y semántica (Higginbotham, 1985). La definición de los predicados carece de la precisión conjuntista de la noción lógica de propiedad y la asignación semántica está lejos de parecerse a la noción de función matemática, demasiado precisa como para explicar satisfactoriamente la relación referencial del lenguaje natural. En este sentido la visión deflacionaria de la referencia (Higginbotham, 2001), aquella que utiliza para su definición nociones como satisfacción o verdad, falla en la explicación de los significados de abundantes oraciones en función de los significados de sus partes y su estructura sintáctica sobre la base de una explicación estrictamente linguiística. Denotación, satisfacción o verdad son términos técnicos de la teoría 
lógica que no tienen contraparte en la semántica natural pues no contamos con una noción de referencia adecuada para la complejidad del lenguaje natural.

El lenguaje natural es un lenguaje humano y la mente, productora del lenguaje, no es una máquina descriptible en términos de la matemática estándar sino un sistema simbólico con su propia lógica en el que no sólo encontramos relaciones deductivas, pues proliferan los desvíos lógicos (Strawson, 1952; Szabó, 2012), y la comprensión de las proferencias sigue procesos tanto deductivos como inductivos y analógicos. Siguiendo a Strawson (1981, p. 685) se puede decir que «hay un desequilibrio conceptual producido por una ceguera colectiva». La regimentación del lenguaje natural en un sistema lógico no implica que el lenguaje natural tenga propiedades lógicas, lo que implica es que el lenguaje natural deja de ser natural. La formalización no es lo que garantiza el proceder científico pues la lógica sólo es un conjunto de procedimientos para el estudio de la inferencia, no tiene una seguridad epistémica especial ni tiene que ver con cómo es el mundo real (Haack, 1978). Los sistemas lógicos sólo pueden explicar las relaciones entre conceptos construidos a través de definiciones estipulativas $\mathrm{y}$, dado que el lenguaje natural no tiene este carácter, usar el lenguaje formal en la descripción del lenguaje natural resulta inadecuado (Frege, 1892; Strawson, 1981; Tarski, 1944). Si uno persiste en tal tarea se enfrenta a un grave dilema: o bien se atiende con seriedad a la verdadera naturaleza de la metafísica que hay detrás del lenguaje, perdiendo con ello el carácter de precisión y utilidad de los sistemas lógicos; o bien se desatiende ésta como medio para preservar la precisión y utilidad de los sistemas lógicos, pero con ello debe uno resignarse a un modo de hablar formal superficial alejado de la naturalidad del lenguaje. La cuestión es que si la semántica trata de lo que el lenguaje habla no se puede llevar a cabo un estudio de la semántica natural sin contar con la ontología de los hablantes, pues en la semántica natural son los hablantes quienes hablan de cosas:

But just as we say that the gardener mows the lawn when we know that it is the lawn-mower which actually does the cutting, so, though we know that the direct relation of symbols is with thought, we also say that symbols record events and communicate facts. (Ogden y Richards, 1923, p. 9).

\section{CONCLUSIONES}

La diferencia entre ambos lenguajes conduce a la conclusión de que el tratamiento formal del lenguaje natural es inadecuado debido a dos razones fundamentales. Por un lado, no podemos dar una definición estipulativa de la ontología necesaria para la asignación semántica en el lenguaje natural tal como se hace en las semánticas formales y, por otro, las nociones semánticas utilizadas en los lenguajes formales están construidas sobre conceptos matemáticos 
que requieren un grado de precisión del que carecen las semánticas naturales. La analogía entre ambos lenguajes con fines semánticos está fundada en una confusión sistemática entre propiedades lógicas y semánticas.

Las teorías veritativo-condicionales se construyen sobre convenciones lógico-matemáticas derivadas de la construcción de lenguajes formales donde verdad y lenguaje coinciden. Sin embargo, en el lenguaje natural hay un hueco insalvable entre ambos. El presupuesto que subyace a las teorías veritativocondicionales es injustificado. La asignación de referencias y de verdad en el lenguaje natural no tiene independencia cognitiva de forma que la interpretación semántica del lenguaje natural exige tener en cuenta el sistema conceptual de los hablantes y la ontología que lo subyace. Tratar el lenguaje natural como un lenguaje formal produce en definitiva dos conclusiones indeseables: o bien su desnaturalización, o bien la construcción de la semántica natural sobre un modo de hablar formal superficial explicativamente vacío.

Los continuos problemas inherentes a la cuestión de la referencia extensional en el lenguaje natural impiden la caracterización misma de la noción de verdad dentro de las teorías veritativo-condicionales pues la determinación de las condiciones de verdad está íntimamente unida a la noción de referencia. Explicar el significado en términos veritativo-condicionales no resuelve el problema del significado sino que nos deja con dos conceptos, verdad y significado (como referencia) señalándose el uno al otro inútilmente (Strawson, 1969). Postular la noción de condiciones de verdad como criterio de significado no es más que cambiar el problema de lugar forzando a explicar en qué consiste la relación de referencia y a entrar en consideraciones metafísicas.

La dirección explicativa lenguaje-realidad falla. No hay una relación de referencia establecida entre palabras y objetos, sino que la relación de referencia está mediada por el sujeto (K. Bach, 2004; Heck, 2011; Seuren, 2009) y, por tanto, la semántica no está comprometida en ofrecer referencias extensionales como base del significado (Chomsky, 2000; Yalcin, 2014). Las teorías extensionales, como las veritativo-condicionales, comenten el error de olvidarse de la cognición y, como afirma Seuren (2009, p. 111), «The extensionalists’ ideal of a mindless logic is thus seen to be illusory». 


\section{REFERENCIAS BIBLIOGRÁFICAS}

BACH, E. W. 1989: Lecciones de semántica formal, tr. N. Bel. Barcelona: Ediciones del Bronce: Edicions de la Universitat de Barcelona, 1997.

BACH, E. W. y CHAO, W. 2012: «The metaphysics of natural language(s)» en R. M. Kempson, T. Fernando y N. Asher (eds.), Philosophy of Linguistics. Oxford: North Holland, 2012, pp. 175-196.

BACH, K. 2004: «Minding the Gap» en C. Bianchi (ed.), The Semantics/Pragmatics Distinction. Palo Alto: CSLI, 2004, pp. 27-44.

BARBA, J. 2010: Lógica, lógicas. Valladolid: Universidad de Valladolid, Secretariado de Publicaciones e Intercambio Editorial.

BIERWISE, M. y SCHREUDER, R. 1992: «From Concepts to Lexical Items», Cognition 42 , pp. 23-60.

CARLSON, G.N. 1977: Reference to Kinds in English. NY: Garland Press.

CHIERCHIA, G. y TURNER R. 1988: «Semantics and Property Theory», Linguistics and Philosophy 11, pp. 261-302.

CHOMSKY, N. 2000: New Horizons in the Study of Language and Mind. Cambridge: Cambridge University Press.

CRESSWELL, M. 1973: Logics and Languages. London: Methuen.

- -,2006: «Formal Semantics» en M. Devitt y R. Hanley (eds.), The Blackwell Guide to the Philosophy of Language. Oxford: Blackwell Publishing, 2006, pp. 131-146.

DAVIDSON, D. 1980: Essays on Action and Events. Oxford: Clarendon Press.

DOWTY, D. R., WALL, R. E. y PETERS, S. 1981: Introduction to Montague Semantics. Dordrecht: D. Reidel Publishing.

FALKUM, I. L. y VICENTE, A. 2015: «Polysemy: Current Perspectives and Approaches», Lingua 157, pp. 1-16.

FREGE, G. 1892: «Sobre sentido y referencia», tr. L. M. V. Villanueva, en L. M. V. Villanueva (ed.), La búsqueda del significado. Lecturas de filosofía del lenguaje. Madrid: Tecnos, 1991, pp. 29-49.

- -,1904: «¿Qué es una función?»,tr. L. M. V. Villanueva, en L. M. V. Villanueva (ed.), Gottlob Frege. Ensayos de semántica y filosofía de la lógica. Madrid: Tecnos, 2013, pp.160-170.

GLANZBERG, M. 2015: «Logical Consequence and Natural Language», en C. Caret y O. Hjortland (eds.), Foundations of Logical Consequence. Oxford: Oxford University Press, pp. 71-120.

HAACK, S. 1978: Filosofía de las lógicas, tr. A. Antón. Madrid: Cátedra, 1982. 
HIGGINBOTHAM, J. 1985: «On Semantics», Linguistic Inquiry 16, pp. 547-593.

— - 2001: «On Referential Semantics and Cognitive Science» en J. Branquinho (ed.), Foundations of Cognitive Science. Oxford: Clarendon Press, 2001, pp. 145-156.

HECK, R. G. 2014: «Semantics and Context-Dependence: Towards Strawsonian Account» en B. Sherman y A. Burgess (eds.), Metasemantics: New Essays on the Foundations of Meaning. Oxford: Oxford University Press, 2014, pp. 327-364.

HORGAN, T. 1986: Psychologism, Semantics and Ontology, Noûs 20 (1), pp. 21-31.

LEPORE, E. 1983: «What Model Theoretic Semantics Cannot Do? », Synthese 54, pp. 167-187.

LINK, G. 1983: «The Logical Analysis of Plurals and Mass Terms» en R. Bäuerle, Ch. Schwarze y A. von Stechow (eds.), Meaning, Use, and Interpretation of Language. Berlin: de Gruyter, 1983, pp. 302-323.

LUDWIG, K. 2012: «Logical Form», en G. Russell y D. Graff (eds.), The Routledge Companion to the Philosophy of Language. NY: Routledge, 2012, pp. 29-41.

MCGEE, V. 2006: «Truth» en M. Devitt y R. Hanley (eds.), The Blackwell Guide to the Philosophy of Language. Oxford: Blackwell Publishing, 2006, pp. 392-410.

OGDEN, C. K. y RICHARDS, I. A. 1923: The Meaning of Meaning. Cambridge: University of Cambridge, 1989.

PEACOCKE, C. 2000: «Theories of Concepts: A Wider Task», European Journal of Philosophy 8 (3), pp. 298-321.

PIETROSKI, P. M. 2005: «Meaning Before Truth» en G. Preyer y G. Peter (eds.), Contextualism in Philosophy: Knowledge, Meaning, and Truth. Oxford: Oxford University Press, 2005, pp. 253-300.

- - 2008: «Semantic Minimalism, Internalist Interpretation», Biolinguistics 2, pp. $317-41$.

PORTNER, P. H. 2005: What is Meaning? Fundamentals of Formal Semantics. Oxford: Blackwell Publishing.

PUTNAM, H. 1975: «The Meaning of 'Meaning'», Minnesota Studies in the Philosophy of Science 7, pp. 131-193.

QUINE, W. V. O. 1953: «Dos dogmas del empirismo», tr. M. Sacristán, en L. M. V. Villanueva (ed.), La búsqueda del significado. Lecturas de filosofía del lenguaje. Madrid: Tecnos, 1991, pp. 245-267.

- - 1956: «Quantifiers and Propositional Attitudes», The Journal of Philosophy 53 (5), pp. 177-187.

RAYO, A. 2013: «A Plea for Semantic Localism», Noûs 47 (4), pp. 647-679. 
ROBINSON, H. 2009: Vagueness, Realism, Language and Thought, Proceedings of the Aristotelian Society 109, pp. 83-101.

RUSSELL, B. 1918: Los problemas de la filosofía, tr. J. Xirau. Barcelona: Labor, 1953.

SCHIFFER, S. 2015: «Meaning and Formal Semantics in Generative Grammar», Erkenntnis 80 (1), pp. 61-87.

SEUREN, P.A.M. 2009: Language from Within I. Language in Cognition. Oxford: Oxford University Press.

STRAWSON, P. F. 1952: Introducción a la teoría lógica, tr. J. Ameller V. Buenos Aires: Editorial Nova, 1969.

—-,1969: «Significado y verdad», tr. L. M. V. Villanueva, en L. M. V. Villanueva (ed.), La búsqueda del significado. Lecturas de filosofía del lenguaje. Madrid: Tecnos, 1991, pp. 356-373.

—-,1981: «Análisis y metafísica descriptiva», tr. J. L. Zofío, en Muguerza (ed.), La concepción analítica de la filosofía, 1986, pp. 597-644.

SZABÓ, Z.G. 2012: «Against Logical Form» en G. Preyer (ed.), Donald Davidson on Truth, Meaning, and the Mental. Oxford: Oxford University Press, 2012.

TARSKI, A. 1944: «The Semantic Conception of Truth», en Martinich (ed.), The Philosophy of Language. Oxford: Oxford University Press, 1996.

YALCIN, S. 2014: «Semantics and Metasemantics in the Context of Generative Grammar» en A. Burgess y B. Sherman (eds.), Metasemantics: New Essays on the Foundations of Meaning. Oxford: Oxford University Press, 2014, pp. 17-54.

Leticia Santos Varona es profesora de filosofía de Educación Secundaria y está realizando su tesis doctoral en el Departamento de Filosofía de la Universidad de Valladolid

Líneas de Investigación:

Filosofía del lenguaje, semántica

Correo electrónico: leticia.santos.varona@alumnos.uva.es 
